\title{
VIVIENDO CON UNA OSTOMÍA: PERCEPCIONES Y EXPECTATIVAS DESDE LA FENOMENOLOGÍA SOCIAL
}

\author{
Augusto Hernán Ferreira Umpiérrez $z^{1}$
}

\footnotetext{
${ }^{1}$ Doctor en Enfermería. Profesor Agregado Departamento de Ciencias de la Enfermería de la Facultad de Enfermería y Tecnologías de la Salud Universidad Católica del Uruguay. Montevideo, Uruguay. E-mail: auferrei@ucu.edu.uy
}

RESUMEN: La finalidad de este estudio fue comprender la experiencia de vida de un grupo de pacientes portadores de colostomía develando sus expectativas en relación al cuidado de enfermería. Se optó por una investigación cualitativa, con el enfoque fenomenológico social de Alfred Schütz, en la cual participaron nueve pacientes portadores de colostomía. Del análisis de las entrevistas, a la luz del referencial, se identificó el tipo vivido de estos sujetos de estudio los cuales mostraron un contexto vivencial en el cual es de relevancia el poder de adaptación y el entorno socio laboral, mostrando expectativas respecto a la enfermera como asesora para la transición, el deseo del cuidado humanizado y la construcción de nuevos horizontes.

DESCRIPTORES: Colostomía. Atención de enfermería. Calidad de vida. Experiencias de vida.

\section{LIVING WITH AN OSTOMY: PERCEPTIONS AND EXPECTATIONS FROM A SOCIAL PHENOMENOLOGICAL PERSPECTIVE}

\begin{abstract}
The aim of this study was to understand the life change events of a group of patients with a colostomy, as well as explore their nursing care expectations. Nine patients with a colostomy participated in this qualitative research, which was based on the social phenomenological approach of Alfred Schütz. The analysis of the interviews, using this frame of reference, identified the type of experience lived by these study subjects, whose life change contexts underscored the importance of the power of adaptation and the social and job environment. It also revealed their expectations regarding nurses as counselors during the transition period, their desire for compassionate care and the building of new horizons.
\end{abstract}

DESCRIPTORS: Colostomy. Nursing care. Quality of life. Life change events.

\section{VIVENDO COM UMA ESTOMIA: PERCEPÇÕES E EXPECTATIVAS À LUZ DA FENOMENOLOGIA SOCIAL}

RESUMO: O objetivo do presente estudo foi compreender a experiência de vida de um grupo de pacientes com colostomía, revelando suas expectativas em relação ao cuidado de enfermagem. Optou-se por uma pesquisa qualitativa com abordagem da Fenomenologia Social de Alfred Schütz. Foram entrevistados nove pacientes com colostomia. A análise das entrevistas, à luz do referencial, identificou o modo de vida dos sujeitos do estudo, que apresentaram um contexto experiencial em que a relevância é o poder de adaptação e o ambiente social e de trabalho, mostrando as expectativas para a enfermeira como consultora para a transição, o desejo do cuidado humanizado e a construção de novos horizontes.

DESCRITORES: Colostomia. Cuidados de enfermagem. Qualidade de vida. Acontecimentos que mudam a vida. 


\section{INTRODUCCIÓN}

La experiencia de vida de los pacientes portadores de una colostomía es un fenómeno que se torna necesario de ser comprendido por los profesionales que forman parte del proceso de atención de enfermería a personas con este tipo de alteraciones. En ese entendido es que este estudio estuvo guiado por las siguientes inquietudes: ¿Cómo es la experiencia de vivir con una ostomía? ¿Cuáles son las expectativas que el paciente tiene respecto al cuidado de enfermería? ¿Cómo se visualiza a futuro?

El abordaje de este tipo de pacientes debe traspasar el ámbito puramente biológico para transitar hacia un enfoque biopsicosocial, donde la enfermera encuentra un campo especial de acción, de modo tal que la consejería y apoyo en la toma de decisiones se torna de relevancia en la atención integral del usuario. ${ }^{1}$

Los resultados del proceso de atención son sustancialmente mejores si se anima al paciente a aceptar su estoma y participar en la vida social, retomando las actividades comunes de su vida diaria. Sin dudas que el reto para los profesionales es encontrar las formas para lograr estos objetivos. ${ }^{2}$

El impacto de una ostomía puede ser devastador, incluso en un período postoperatorio sin complicaciones. El paciente se encuentra en ocasiones sin conocimiento previo, con un segmento del intestino exteriorizado a través de la pared abdominal. Se suceden una serie de vivencias que generan dificultades de adaptación, donde la depresión resulta común. ${ }^{3}$ A esto, se suma que las estancias hospitalarias más cortas que tienen lugar en la actualidad, sobre todo donde hay programas de alta precoz, hacen que el paciente tenga escaso tiempo para adaptarse y ser competente para el autocuidado de su estoma al alta hospitalaria. ${ }^{4}$

Por lo expuesto, la enfermera se constituye en un profesional fundamental para apoyar al paciente ostomizado hasta que se considere competente para asumir él mismo, o en su defecto, su familia, el cuidado de su estoma. Es fundamental entender los hábitos del paciente, sus percepciones y actitudes en relación a los demás, sentimientos y emociones demostrados en las más diversas situaciones. ${ }^{5}$ Por lo tanto, tener en cuenta sus valores y sentimientos, así como la integración de la familia, forma parte de los aspectos básicos del abordaje de este tipo de pacientes. ${ }^{6}$

En esa línea, como muestran estudios sobre el tema, ${ }^{5-7}$ se torna necesaria la comprensión de este fenómeno por parte del profesional que brinda cuidados, el cual colabora con el mejoramiento de la calidad de vida, abordando al paciente y su familia desde el período preoperatorio. ${ }^{8}$

El abordaje y la resolución de este tipo de problemas de salud dependen de los recursos del paciente, así como también de los profesionales que lo atienden y de la estructura de atención ofrecida. En ese contexto, la comprensión del sujeto se torna de relevancia para ofrecer una atención de calidad, brindando educación enmarcada en una estrategia pedagógica que colabora para que el usuario ejerza su condición de sujeto independiente y autónomo. ${ }^{9}$

Por lo anteriormente expuesto, y tratando de develar las inquietudes relacionadas a este fenómeno, el objetivo de este estudio fue definido como: comprender la experiencia de vida de un grupo de pacientes portadores de colostomía develando sus expectativas en relación al cuidado de enfermería.

\section{METODOLOGÍA}

Se trata de un estudio cualitativo, basado en la premisa de que los conocimientos sobre los individuos son solo posibles con una descripción de la experiencia humana, como ella es vivida y tal como ella es definida por sus actores. ${ }^{10-11}$ Se optó por el método fenomenológico ${ }^{12}$ como referencial teórico-filosófico, de manera de comprender la realidad del día a día en la cual estamos inmersos los sujetos. ${ }^{13}$

El enfoque elegido fue el de la Fenomenología Social de Alfred Schütz, el cual ofrece un método sistemático para comprender mejor los aspectos sociales de la acción humana. Dicho enfoque ofrece un camino que articula conceptos de intencionalidad, intersubjetividad, acciones humanas, relacionamiento social, expectativas y otros que pueden lograr una mejor comprensión del mundo social de las personas. ${ }^{12}$ La comprensión del fenómeno, como forma vivida en la cotidianeidad, supone un análisis del comportamiento social en relación a los motivos y finalidades. Schütz distingue dos tipos de motivos en el comportamiento social: los que envuelven la acción y la finalidad, son los "motivos en vista de..." o "motivos para", y los que suponen el escenario de los actores, el medio ambiente, la disposición psíquica, son los "motivos debidos a..." o "motivos porque". ${ }^{14}$

Conceptos claves de este referencial son algunos como la intersubjetividad, basándose en que nuestro mundo está compuesto por semejantes. 
Este mundo no se da en la esfera de lo privado, sino que existe en una intersubjetividad compartida, donde las vivencias son interpretadas recíprocamente. Otro concepto es el de la relación cara a cara, dado que siempre hay otra persona que está al alcance de nuestra experiencia directa cuando comparte un espacio y un tiempo. Su experiencia fluye al lado de la nuestra y se entabla una relación entre dos. Las actitudes están influidas por el acervo de conocimientos y la situación biográfica de cada sujeto, dado que las experiencias vividas anteriormente son vivencias almacenadas y son las que determinarán los elementos relevantes de la acción. Estos aportes son sustanciales para la comprensión de los procesos de la interacción enfermera - paciente contribuyendo a las mejoras del ejercicio profesional. ${ }^{15}$

En esa línea, el mundo vivido por la persona es, desde el principio, un mundo social y cultural dentro del cual el sujeto se relaciona. El conocimiento del mismo es para fines prácticos, donde la comprensión de sus conductas, sus motivos, objetivos y planes se originan en circunstancias biográficamente determinadas. ${ }^{16}$

Permeado por estos conceptos, el estudio fue realizado en la ciudad de Montevideo, Uruguay, participando nueve pacientes portadores de colostomía, de ambos sexos, con edades entre 49 y 72 años. De ellos tres portaban una colostomía temporaria y seis una colostomía definitiva. Todos ellos, con tres meses o más de haber sido intervenidos quirúrgicamente, fueron seleccionados cuidadosa e intencionalmente, de una base de datos conocida por el investigador, por sus posibilidades de ofrecer información profunda y detallada sobre el asunto de interés para la investigación. ${ }^{17} \mathrm{La}$ recolección de los datos se realizó entre febrero y mayo de 2012, mediante entrevistas en profundidad, las cuales fueron establecidas de común acuerdo con los sujetos participantes, garantizando el anonimato, la privacidad y la confidencialidad de la información entregada. Se respetaron las consideraciones éticas establecidas en el Decreto n. 379/008 del país ${ }^{18}$ para las investigaciones con seres humanos, informándose a los participantes los objetivos del estudio, sus alcances, y todos los aspectos relativos al consentimiento libre e informado. ${ }^{19}$ El estudio fue autorizado por el Comité de Ética Asesor de la Facultad de Enfermería y Tecnologías de la Salud de la Universidad Católica del Uruguay, con el número de protocolo n. 004/2012.

Dado el tipo de estudio, el número de participantes no fue definido previamente, habién- dose cerrado la recolección de los datos a partir del momento en que las inquietudes del estudio fueron respondidas y los objetivos alcanzados, comenzándose a tornar repetitivos, ${ }^{17}$ mostrando las señales de develación del fenómeno. ${ }^{20}$ Los sujetos fueron identificados por códigos para respetar su anonimato, identificando los discursos con la letra E (de Entrevista), seguida de un número secuencial del 1 al 9.

Las entrevistas fueron guiadas por las preguntas orientadoras del estudio, las cuales fueron: ¿Cómo es la experiencia de vivir con una ostomía? ¿Cuáles son tus expectativas respecto al cuidado de enfermería ¿Cómo te visualizás de ahora en adelante? Estas preguntas fueron definidas a la luz del referencial teórico-filosófico elegido, buscando que fueran una guía para la develación del fenómeno en cuestión. Las entrevistas fueron grabadas en un dispositivo electrónico y posteriormente fueron transcriptas. Mediante su transcripción exacta, se aseguró la integralidad del proceso y la reducción fenomenológica que busca llegar a la esencia de las formas que componen esa experiencia psíquica del otro. ${ }^{11}$

El análisis de las entrevistas siguió los pasos propuestos sobre métodos de análisis cualitativo de los datos ${ }^{21} \mathrm{y}$ de investigadores de la fenomenología social ${ }^{22}$ que consiste en: lectura atenta de cada entrevista, para aprehender el sentido global de la experiencia vivida por cada sujeto; agrupamiento de los aspectos significativos de las entrevistas para la composición de las categorías concretas; análisis de estas categorías, buscando la expresión de la vivencia de los pacientes portadores de colostomía; discusión de los resultados a la luz de la Fenomenología Social de Alfred Schütz y de otras referencias relacionadas al tema. ${ }^{23}$ Las categorías concretas fueron compuestas por descripciones que mostraran de forma expresiva la vivencia de los sujetos, nominándolas al encontrar convergencias a la luz del referencial.

Se buscó comprender la experiencia de los sujetos en el entendido de que el cuidado comprensivo dinamiza este proceso y estimula la movilización del sujeto desde la parálisis que provoca la condición desfavorable de salud, hacia acciones que abren nuevas posibilidades para su bienestar. ${ }^{24}$

\section{RESULTADOS}

El estudio riguroso de las entrevistas realizadas permitió que emergieran cinco categorías representativas de la experiencia de vida y expec- 
tativas de los sujetos de estudio, las cuales fueron nominadas como: el poder de adaptación, el entorno socio laboral, la enfermera como asesora para la transición, el deseo de cuidado humanizado, y construcción de nuevos horizontes.

Considerando los principales conceptos del referente filosófico se pudo observar como en cada categoría emergieron los "motivos porque" (contextos de motivación) y los "motivos para" (proyectos y expectativas), a la luz del referencial fenomenológico social. Se presenta, a continuación, trechos de entrevistas que dan lugar a las categorías conformadas.

\section{El poder de adaptación (motivos porque)}

Los sujetos del estudio muestran en sus discursos que la experiencia se construye desde las capacidades de adaptación a la nueva forma de vida. Es de relevancia para ellos, en mayor o menor medida, poder adaptarse a la nueva relación desde lo que cada uno trae consigo:

[...] tengo que adaptarme, quiera o no tengo que hacerlo. No es fácil porque yo nunca había pasado por esto, obviamente, ni conocía nada de esto, nadie, ni un conocido, ni un vecino siquiera. Es difícil pero sino te adaptás marchás (E2).

[...] yo sé que tengo fuerza, siempre la he tenido, esa fuerza que Dios me da, y tengo que acostumbrarme a vivir con esto. Ayer mismo hablaba con mi señora y le decía que no se haga problema que yo puedo. Ya pedí que me cambien de lugar en mi trabajo y me armé un bolso con las cosas que necesito para manejarme lo mejor posible (E6).

[...] para mí es difícil, yo sé que tengo que acostumbrarme, arreglar mis cosas, acostumbrarme a una nueva forma de vida. Ahora no es como antes que uno salía cuando quería y sin tener en cuenta nada, ahora tengo que aprender a vivir con esto (E8).

\section{El entorno socio laboral (motivos porque)}

De las entrevistas emergen datos referidos al contexto socio laboral de los sujetos, el cual denota especial importancia para la evolución del paciente y la aceptación de su problema.

Desde esa experiencia, de lo social y del entorno del trabajo, se vivencia para salir adelante:

[...] sino hubiera sido por mi familia yo me muero, no estaría acá. Mi esposa encaró todo y me sacó adelante, mi hija mayor también, porque la otra está en España y no puede estar acá [...]. Tengo un vecino que ya había pasado por esto y para mí fue un apoyo fundamental (E6).

[...] yo creo que si tenés ayuda es más fácil, en la mutualista a mí me dieron una mano enorme. Las enfermeras ayudaban a mi esposa, le explicaban, le dijeron todo [...]. En mi trabajo me dieron una mano también, pero yo que sé, parece que todos hablan de vos, no sabés si te ayudan bien o te tienen lástima [...] vivir con esto [...] que se yo [...] es bravo salir a la calle y no estar pensando que te sale olor o en el trabajo más todavía, pero dicen que no larga olor (E5).

\section{La enfermera como asesora para la transición (motivos para)}

En la reducción de los datos emerge la figura de la enfermera fundamentalmente como asesora del proceso, siendo una pieza fundamental para la transición hacia una nueva vida.

Emergen las expectativas con respecto a la relación enfermera-paciente:

[...] la verdad que yo lo que espero es que me atiendan bien, uno está con muchos miedos, uno no conoce lo que le pasa y nunca vivió esto entonces si tenés a alguien que te explique, que te diga cómo hacer las cosas, cómo encarar, es mucho más fácil y te quedás más tranquilo [...] acá viene siempre una nurse que te explica todo, me dio cosas escritas, va, a mi esposa le dio porque yo al principio no quería saber de nada (E4).

[...] y yo que sé, a mi me atendieron bien [...] ¿Expectativas? [...] y tengo bastantes, que me diga cómo hacerme esto, aunque ahora ya sé, la nurse me explicó como tengo que cambiarme el aro y que tengo que hacer si se me despega [...] me asesoró en todo y si tengo alguna duda la llamo y listo [...] es muy importante para mí tener quien me ayude a encaminarte en esto tan nuеvo (E8).

[...] esto es como meterte en un túnel [...] si tenés quien te guíe es lo mejor, alguien que conozca el camino $y$ te ayude a salir a luz (E9).

\section{El deseo de cuidado humanizado (motivos para)}

Como expectativa emergente de los discursos surge el deseo de ser atendido en forma humana, cercana, con calidez. Es una de los aspectos que más esperan los sujetos del estudio:

[...] para mí es fundamental que te den corte, que te atiendan bien, que te saluden, que sean humanas, porque viste que a veces uno ve que tienen mucho trabajo y yo las comprendo, pero uno está en esta situación tan 
rara que uno precisa un mimo a veces. Acá las enfermeras vienen y uno está esperando a veces porque ya sabe que son más atentas que el médico, hablan más claro [risa] (E1).

[...] mi hija dice que les pregunte a las enfermeras que hablan en criollo [risa], no, fuera de broma, eso es lo que uno espera, que sean cálidas, ya bastante tiene uno como para tener más problemas [...] yo espero que me traten como en casa (E5).

[...] mis expectativas son las de todos los pacientes me parece, que te traten bien, que te comprendan. A veces no es que uno no quiera hacer lo que te dicen, es que uno está como rebelde, entonces esperás comprensión, que se yo, uno no es una máquina, al final uno es un ser humano y precisa que sean humanos con uno (E8).

\section{Construcción de nuevos horizontes (motivos para)}

Los datos emergidos de las entrevistas muestran que los sujetos se visualizan en nuevos horizontes, construcciones a futuro que surgen de la develación del fenómeno:

[...] y ahora hay que enfrentar la cosa, me decía la nurse que yo puedo hacer todo lo que hacía antes, hasta ir a la playa me dijo, yo no sé, pero si es así, es un lujo que no pensaba darme, tengo que estar bien, mi hija quiere eso y eso voy a hacer (E2).

[...] tengo tantos planes [...] uno de ellos es disfrutar de mi nieta, ahora más que nunca luego de haber pasado por esto, la vida hay que disfrutarla porque se te va volando de un momento a otro (E5).

[...] de ahora en adelante quiero volver al trabajo, no va a ser lo mismo, pero tengo que volver lo mejor posible. Me dijeron que puedo hacer todo lo que quie$\mathrm{ra}$, bueno, con algunos límites obviamente, pero vida normal, o casi normal (E6).

[...] ya me adapté. La enfermera me dijo que ahora tengo que ir a hacer el trámite antes de irme. Pero adaptarme ya está [...] me visualizo en casa con mis nietos que ya les expliqué como es esto que tengo (E9).

\section{DISCUSIÓN}

La experiencia de vida de los sujetos del estudio deja en evidencia algunos aspectos relacionados a su contexto de motivación, así como otros respecto a sus expectativas y a como se visualizan a futuro. El análisis de las entrevistas, a la luz del referencial teórico-filosófico, permitió develar los "motivos porque" y los "motivos para". Se ha mostrado la manera en la cual experiencian el mundo cotidiano, desde el enfoque fenomenológico social. ${ }^{25}$

Las categorías emergidas, relacionadas a los "motivos porque", dan cuenta del bagaje con el que los sujetos cuentan, desde donde construyen su realidad, mostrando que los sujetos resaltan el poder de adaptación y el entorno socio laboral.

En relación al poder de adaptación destacamos que es construido desde lo que cada persona trae, teniendo como punto de partida las circunstancias de situación biográfica, el acervo de conocimientos y las experiencias vividas anteriormente. ${ }^{15-16} \mathrm{La}$ ausencia de conocimiento previo de este tipo de situaciones tornan a las personas susceptibles, vivenciando la falta de adaptación y la depresión como resultados comunes. ${ }^{3}$ Desde nuestro punto de vista, y de acuerdo con los autores, el rol de la enfermera es fundamental para facilitar este proceso de adaptación, asistiendo, educando y conteniendo al paciente y a su familia, favoreciendo el equilibrio con su entorno. ${ }^{26}$

En la línea de lo anterior surge como elemento destacado el entorno socio laboral. La relación con los otros, permeada de intersubjetividad, denota un mundo compartido con semejantes. ${ }^{12}$ Los datos emergidos de las entrevistas muestran el lugar destacado que los sujetos otorgan a la familia y los amigos, así como la relevancia de la enfermera en el proceso de acompañamiento. Esto coincide con los aportes de la fenomenología de Schütz, donde se destaca la relación cara a cara desde la concepción de que siempre hay otras personas que están al alcance de nuestra experiencia de vida y que comparten nuestro espacio. ${ }^{16}$ También, los datos se muestran acordes a estudios donde se insiste en que el reto de quienes comparten un espacio con estos pacientes pasa por encontrar formas para estimular su participación en la vida social, ${ }^{2}$ donde el profesional de enfermería emerge como una figura de apoyo a la transición hacia las nuevas experiencias.

En ese sentido, los sujetos mostraron expectativas claras, develándose los "motivos para" del enfoque, visualizando a la enfermera como asesora para la transición, mostrando el deseo de cuidado humanizado y la construcción de nuevos horizontes que incluyen a su familia y su entorno.

La comprensión de las expectativas del paciente colabora con las mejoras de la práctica de la enfermería, impregnándose la relación de ayuda de intersubjetividad y espacios compartidos, ${ }_{1}^{14}$ para ofrecer un cuidado comprensivo. ${ }^{24}$ Las personas con una colostomía deben vivir la experiencia de aceptarse como un ser diferente, requiriendo 
un ajuste de su imagen y auto-concepto. ${ }^{27}$ En ese proceso, la enfermera, mediante la relación de ayuda, acompaña y educa. Los datos emergidos encuentran concordancia con autores que resaltan la figura de la enfermera como asesora, consultora y gestora de cuidados en la relación terapéutica. ${ }^{1-5}$

Los deseos mostrados de un cuidado humanizado es un aspecto resaltado por todos los sujetos. Las necesidades de una atención cálida y cercana permean las entrevistas realizadas en este estudio. El develar esta necesidad por encima de otras, que podrían ser de igual o mayor preocupación del paciente, denota la importancia que supone la comprensión del fenómeno de estudio, dando insumos para abordar al ser humano desde todas sus dimensiones, de acuerdo a estudios que destacan los valores y sentimientos de las personas $^{5-8}$ impregnando el cuidado y la interacción enfermera-paciente del componente humano, dejando de lado el centrarse en el modelo biologicista que traspasa las instituciones sanitarias.

Por último, no es menor la proyección que hacen los sujetos a futuro construyendo nuevos horizontes, visualizándose en un mundo compartido, que supone enfrentar los nuevos estilos de vida que pueda suponer portar una ostomía. En todos los casos, emerge el deseo de un mundo con los otros, envuelto de relacionamiento social, en principio con el entorno familiar más cercano, lo cual encuentra base en los conceptos que permean el enfoque teórico-filosófico de este estudio. ${ }^{12,14,16,25}$

\section{CONCLUSIÓN}

Orden a lo expuesto, encontramos que se ha mostrado una parte del fenómeno estudiado, habiéndose visualizado aspectos que colaboran con las mejoras de la práctica del cuidado enfermero en relación a pacientes portadores de colostomía.

Los hallazgos encontrados y analizados a la luz de la teoría permiten visualizar las implicancias que tienen para la relación establecida entre enfermera, paciente y familia. El cuidado de enfermería y su gestión es deseable que estén dirigidos a que la enfermera se empodere en su rol de asesora, atendiendo especialmente a favorecer la adaptación del paciente a su nuevo modo de vida, rescatando y potenciando sus fortalezas, y colaborando en la superación de sus debilidades. La gestión del cuidado, relacionada a la reinserción en el entorno social y laboral del paciente, debe ser otro objetivo del plan de cuidados, a la vez de establecer acciones dirigidas a un cuidado huma- nizado, holístico, que deje de estar centrado en la reducción biologicista. A su vez, como asesora en el proceso de transición, la enfermera forma parte del contexto del paciente, colaborando con él y su familia en la construcción de los nuevos desafíos a los que se enfrenta.

Al concluir, manifestamos que este estudio se limita a un campo determinado, dado que no ofrece posibilidades de generalización por el tipo de método con el que se abordó. Se buscaron las particularidades de los sujetos, conociendo cada vivencia en profundidad, no siendo objeto de este estudio la generalización de los resultados. Sin embargo, los aportes que han surgido entendemos que son un insumo importante para la comprensión de los procesos de salud de este tipo de pacientes, dejando caminos abiertos para realizar nuevos estudios que enriquezcan el conocimiento sobre el tema, aportando a generar evidencias que colaboren con las mejores prácticas en la relación de ayuda que tiene lugar en la interacción enfermera-paciente.

\section{REFERENCIAS}

1. Ferreira A. Proceso de atención al paciente ostomizado: gestión de cuidados integrales. Montevideo (UY): Ed. Psicolibros Waslala; 2011.

2. Kingsley L, Simmons J. Adjustment to colostomy: stoma acceptance, stoma care self-efficacy and interpersonal relationships. J Adv Nurs. 2011 Dic; 60(6):627-35.

3. Constantino L, Lázaro E, Sosa M. Problemática actual del paciente con ostomia. Rev Cubana Med Milit. 2001 Nov; 30(4):256-61.

4. Black P. Stoma care nursing management: cost implications in community care. Br J Community Nurs. 2009 Aug; 14(8): 350-5.

5. Arrais F, Souza P, Leite T, Giménez M. Assistência de enfermagem a paciente com colostomia: aplicação da teoria de Orem. Acta Paul Enferm. 2008 Mar; 21(1):94-100.

6. Maruyama S, Zago M. O processo de adoecer do portador de colostomia por câncer. Rev Latino-Am Enfermagem. 2005 Abr; 13(2):216-22.

7. Bellato R, Tao S, Moraes C, Castro P. A condição crônica e as repercussões que traz para a vida da pessoa e sua família. Cienc Cuid Saude. 2007 JanMar; 6(1):40-50.

8. Bachelet V, Collet $\mathrm{G}$, Ribal A. The role of the stoma therapy nurse in the preoperative period. Rev Infirm. 2012 May; (181):25-6.

9. Alvarenga P, Neide A. Perspectiva educativa do cuidado de enfermagem sobre a manutenção da estomia de eliminação. Rev Bras Enferm. 2011 Apr; 64(2):322-7. 
10. Polit D, Beck CT, Hungler F. Fundamentos da pesquisa em enfermagem: avaliação de evidências para a prática da enfermagem. $7^{\mathrm{a}}$ ed. Porto Alegre (RS): Artes Médicas; 2011.

11. Martins J, Bicudo MAV. A pesquisa qualitativa em psicologia: fundamentos e recursos básicos. $5^{\mathrm{a}} \mathrm{ed}$. São Paulo (SP): Centauro; 2005.

12. Muñoz LA, Lorenzini A. La fenomenología en la producción de conocimientos de Enfermería. En: Prado M, Souza M, Carraro T. Investigación cualitativa en enfermería: contexto y bases conceptuales. Washington D. C. (US): OPS; 2008.

13. Terra M, Silva L, Camponogara S, Santos E, Souza A, Erdmann A. Na trilha da fenomenologia: um caminho para a pesquisa em enfermagem. Texto Contexto Enferm. 2006 Ou-Dez; 15(4):672-8.

14. Schütz A. Estudios sobre teoria social: escrito II. Buenos Aires (AR): Amorrortu; 2003.

15. Camatta M, Nasi C, Schaurich D, Schneider J. Contribuciones de la sociología fenomenológica de Alfred Schütz para las investigaciones en enfermería. Braz J Nurs. 2008 Apr; 7(2):78-98.

16. Vollrath A, Angelo M, Muñoz L. Vivencia de estudiantes de enfermería de la transición a la práctica profesional: un enfoque fenomenológico social. Texto Contexto Enferm. 2011; 20(spe): 66-73.

17. Martínez-Salgado C. El muestreo en investigación cualitativa: principios básicos y algunas controversias. Ciênc Saúde Coletiva [Internet]. 2012 Mar [cited 2012 Aug 22]; 17(3):613-9. Available from: http:/ / www.scielo.br/scielo.php?script=sci_ arttext\&pid=S1413-81232012000300006\&lng=en

18. Uruguay. Decreto de Ley n. 379/008. Investigaciones con seres humanos. Diario Oficial $n^{\circ} 4573 / 08$, diciembre de 2008.

19. Nunes E. Ethical aspects considered by researchers who use qualitative approaches in health. Ciênc Saúde Coletiva [online]. 2008 Apr [cited 2012 Aug 22];
13(2): 351-60. Available from: http:/ / www.scielosp. org / scielo.php? script=sci_arttext\&pid=S141381232008000200010\&lng=en

20. Noreña A, Alcaraz-Moreno N, Rojas J, Rebolledo D. Aplicabilidad de los criterios de rigor y éticos en la investigación cualitativa. Aquichán Col. 2012 Dic; 12 (3):213-27.

21. Lessard-Hérbert M, GoyetteG, Boutin G. Investigação cualitativa, fundamentos e prácticas. $4^{\mathrm{a}} \mathrm{ed}$. Portugal: Stória Editores, Instituto Piaget; 2010.

22. Merighi MAB, Jesus MCP, Domingos SRF, Oliveira DM, Baptista PCP. Being a nursing teacher, woman and mother: showing the experience in the light of social phenomenology. Rev Latino-Am Enfermagem. [online] 2011 Jan-Feb [acesso 14 Out 2011]; 19(1):164-70. Available from: http://www. scielo.br/pdf/rlae/v19n1/22.pdf

23. Arias MM, Giraldo CV. El rigor científico en la investigación cualitativa. Invest Educ Enferm. 2011 Nov; 29(3):500-14.

24. Rivera M, Herrera L. Fundamentos fenomenológicos para un cuidado comprensivo de enfermería. Texto Contexto Enferm. 2006; 15(spe):158-63.

25. Wagner HR, organizador. Fenomenología e relaçoes sociais: textos escolhidos de Alfred Schütz. Río de Janeiro (RJ): Zahar; 1979.

26. Coelho $S$, Mendes I. Da pesquisa à prática de enfermagem aplicando o modelo de adaptação de Roy. Esc Anna Nery [online]. 2011 Dec [cited 2012 Aug 22]; 15(4): 845-50. Available from: http:/ / www. scielo.br/scielo.php?script=sci_arttext\&pid=S141481452011000400026\&lng=en

27. Souza PCM, Costa VRM, Maruyama SAT, Costa ALRC, Rodrigues AEC, Navarro. As repercussões de viver com uma colostomia temporária nos corpos: individual, social e político. Rev Eletr Enferm [online]. 2011 Jan-Mar [acesso 2013 Fev 21]; 13(1):509. Disponível em: http://dx.doi.org/10.5216/ree. v13i1.7928. 